\title{
Learning mutational graphs of individual tumour evolution from single-cell and multi-region sequencing data
}

\author{
Daniele Ramazzotti ${ }^{1}$, Alex Graudenzi ${ }^{2,3^{*}}$ (D), Luca De Sano², Marco Antoniotti ${ }^{2,4}$ and Giulio Caravagna ${ }^{5}$
}

\begin{abstract}
Background: A large number of algorithms is being developed to reconstruct evolutionary models of individual tumours from genome sequencing data. Most methods can analyze multiple samples collected either through bulk multi-region sequencing experiments or the sequencing of individual cancer cells. However, rarely the same method can support both data types.

Results: We introduce TRalT, a computational framework to infer mutational graphs that model the accumulation of multiple types of somatic alterations driving tumour evolution. Compared to other tools, TRalT supports multi-region and single-cell sequencing data within the same statistical framework, and delivers expressive models that capture many complex evolutionary phenomena. TRalT improves accuracy, robustness to data-specific errors and computational complexity compared to competing methods.

Conclusions: We show that the application of TRalT to single-cell and multi-region cancer datasets can produce accurate and reliable models of single-tumour evolution, quantify the extent of intra-tumour heterogeneity and generate new testable experimental hypotheses.
\end{abstract}

Keywords: Single-tumour evolution, Single-cell sequencing, Multi-region sequencing, Mutational graphs, Cancer evolution, Tumour phylogeny

\section{Background}

Sequencing data from multiple samples of single tumours can be used to investigate Intra-Tumor Heterogeneity (ITH) in light of evolution [1-3]. Motivated by this observation, several new methods have been developed to infer the "evolutionary history" of a tumour from sequencing data. According to Davis and Navin, there are three orthogonal ways to depict such history [4]: (i) with a phylogenetic tree that displays input samples as leaves [5], (ii) with a clonal tree of parental relations between putative cancer clones [6-9], and (iii) with the order of mutations that accumulated during cancer growth [10-12]. Ideally, the order of accumulating mutations should match the clonal lineage tree in order to reconcile these inferences. Consistently with earlier works of us [13-18],

\footnotetext{
*Correspondence: alex.graudenzi@unimib.it

${ }^{2}$ Dipartimento di Informatica, Sistemistica e Comunicazione, Università degli Studi di Milano-Bicocca, Viale Sarca 336, 20126 Milan, Italy

${ }^{3}$ Institute of Molecular Bioimaging and Physiology of the Italian National Research Council (IBFM-CNR), Viale F.lli Cervi 93, 20090 Segrate, Milan, Italy Full list of author information is available at the end of the article
}

we here approach the third problem ("mutational ordering") from two types of data: multi-region bulk and singlecell sequencing.

Bulk sequencing of multiple spatially-separated tumour biopsies returns a noisy mixture of admixed lineages [19-23]. We can analyse these data by first retrieving clonal prevalences in bulk samples (subclonal deconvolution), and then by computing their evolutionary relations [24-31]. Subclonal deconvolution is usually computationally challenging, and can be avoided if we can read genotypes of individual cells via single-cell sequencing (SCS). Despite this theoretical advantage, however, current technical challenges in cell isolation and genome amplification are major bottlenecks to scale SCS to whole-exome or whole-genome assays, and the available targeted data harbours high levels of allelic dropouts, missing data and doublets [32-35]. Thus, the direct application of standard phylogenetic methods to SCS data is not straightforward, despite being theoretically viable [36]. Notice

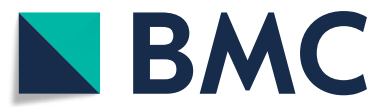

(C) The Author(s). 2019 Open Access This article is distributed under the terms of the Creative Commons Attribution 4.0 International License (http://creativecommons.org/licenses/by/4.0/), which permits unrestricted use, distribution, and reproduction in any medium, provided you give appropriate credit to the original author(s) and the source, provide a link to the Creative Commons license, and indicate if changes were made. The Creative Commons Public Domain Dedication waiver (http://creativecommons.org/publicdomain/zero/1.0/) applies to the data made available in this article, unless otherwise stated. 
that a common feature of most methods for cancer evolution reconstruction is the employment of the Infinite Sites Assumption (ISA), together with the assumption of no back mutation [24-35], even though recent attempts (e.g., [9]) have been proposed to relax such assumption in order to model relevant phenomena, such as convergent evolutionary trajectories [37].
In this expanding field, we here introduce TRalT (Temporal oRder of Individual Tumors - Figs. 1 and 2), a new framework for the inference of models of singletumour evolution, which can analyse, separately, multiregion bulk and single-cell sequencing data, and which allows to capture many complex evolutionary phenomena underlying cancer development. Compared to other

A Tumor phylogeny (mutational ordering)

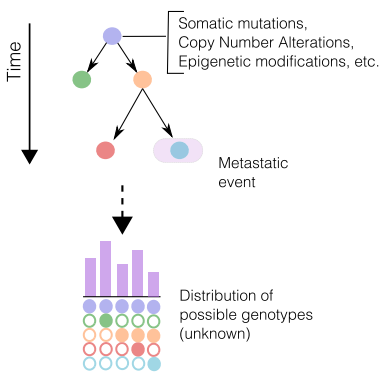

B Multi-region bulk sequencing

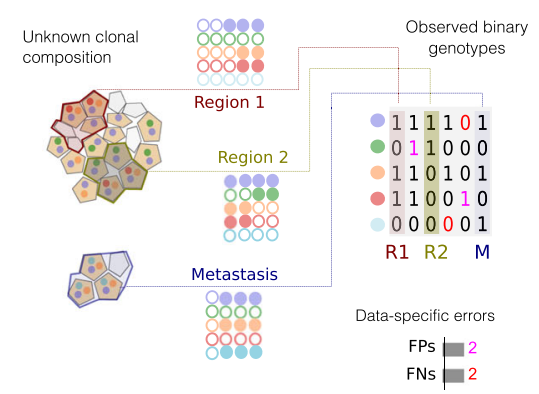

D Scenarios of tumor evolution
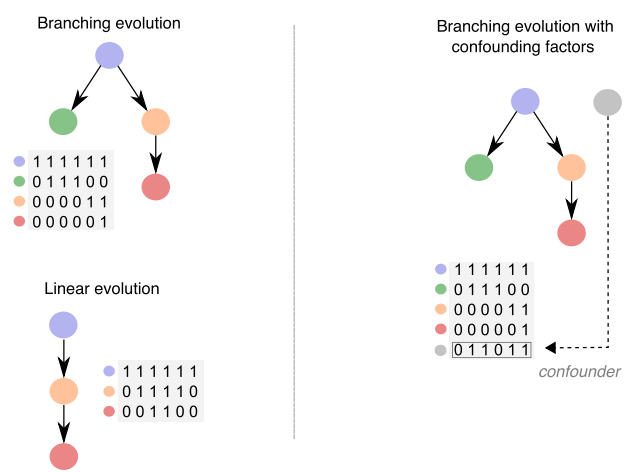

C Single-cell sequencing

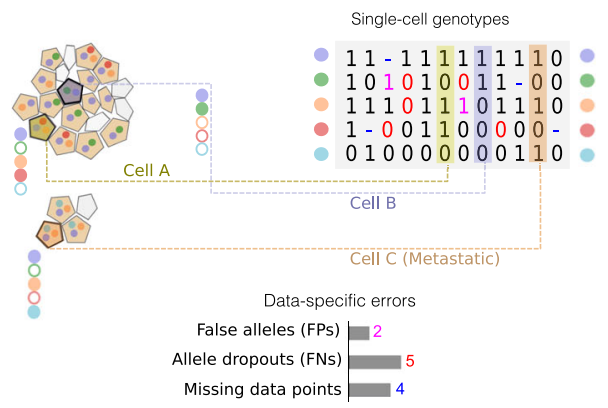

-cell genotypes

$101010011-00$

111011101110

$1-001100000-0$

Missing data points $\quad 4$

Fig. 1 a A tumour phylogeny describes the order of accumulation of somatic mutations, CNAs, epigenetic modifications, etc. in a single tumour. The model generates a set of possible genotypes, which are observed with an unknown spatial and density distribution in a tumour (primary and metastases). b Multi-region bulk sequencing returns a mixed signal from different tumour subpopulations, with potential contamination of non-tumour cells (not shown) and symmetric rates of false positives and negatives in the calling. Thus, a sample will harbour lesions from different tumour lineages, creating spurious correlations in the data. c If we sequence genomes of single cells we can, in principle, have a precise signal from each subpopulation. However, the inference with these data is made harder by high levels of asymmetric noise, errors in the calling and missing data. d Different scenarios of tumour evolution can be investigated via TRalT. (i) Branching evolution (which includes linear evolution), (ii) Branching evolution with confounding factors annotated in the data, (iii) Models with multiple progressions due to polyclonal tumour origination, or to the presence tumour initiating event missing from input data 


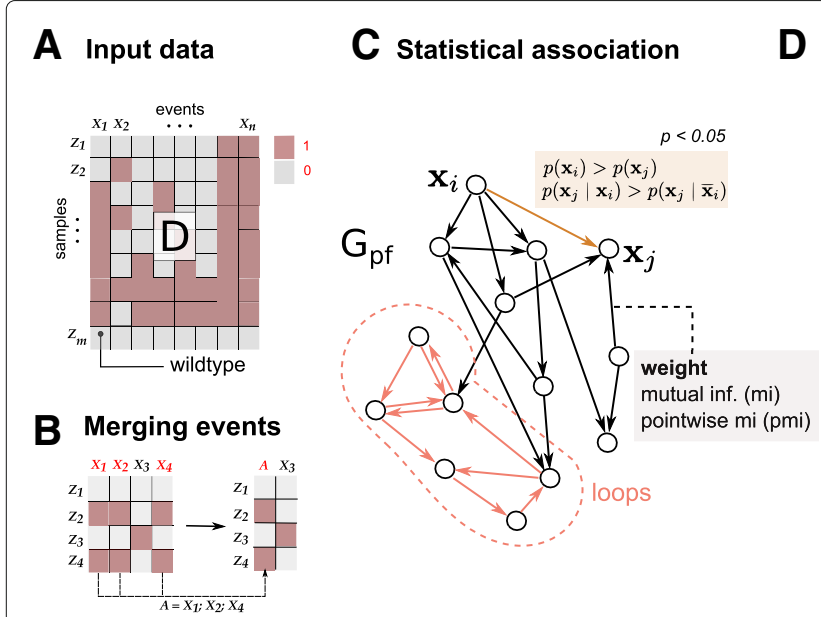

D Loop removal

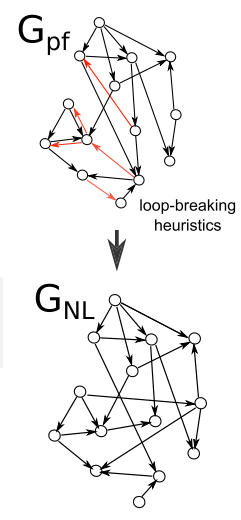

E Edmonds

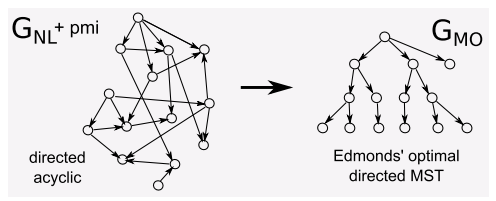

F Chow-Liu

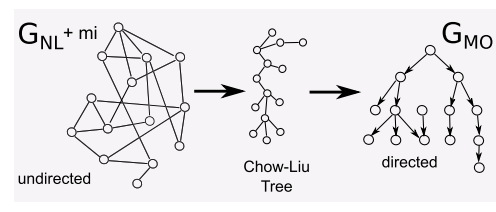

Fig. 2 a TRalT processes a binary matrix $D$ that stores the presence or absence of a variable in a sample (e.g., a mutation, a CNA, or a persistent epigenetic states). b. TRalT merges the events occurring in the same samples ( $\mathbf{x}_{1}, \mathbf{x}_{2}$ and $\mathbf{x}_{4}$, merged to A), as the statistical signal for their temporal ordering is undistinguishable. The final model include such aggregate events. c. We estimate via bootstrap the prima facie ordering relation that satisfies Suppes' conditions (Eq. 1) for statistical association. This induces a graph GpF over variables $\mathbf{x}_{i}$, which is weighted by information-theoretic measures for variables' association such as mutual information or pointwise mutual information. $\mathbf{d}$ TRalT employs heuristic strategies to remove loops from $G_{P F}$ and produce a new graph $G_{N L}$ [14]. e Edmonds's algorithm can be used to reconstruct the optimal minimum spanning tree $G_{M O}$ that minimises the weights in $G_{N L}$; here we use point-wise mutual information (pmi). f. Chow-Liu is a Bayesian mode-selection strategy that computes an undirected tree as a model of a joint distribution on the annotated variable. Then, we provide edge direction (temporal priority), with Suppes' condition (Eq. 1) on marginal probabilities. Therefore, confluences are possible in the output model $G_{M O}$ in certain conditions

approaches that might scale poorly for increasing sample sizes, our methods show excellent computational performance and scalability, rendering them suitable to anticipate the large amount of genomic data that is becoming increasingly available.

\section{Results}

TRalT is a computational framework that combines Suppes' probabilistic causation [38] with information theory to infer the temporal ordering of mutations that accumulate during tumour growth, as an extension of our previous work [13-18]. The framework comprises 4 algorithms (EDMONDS, GABOW, CHOWLIU and PRIM) designed to model different types of progressions (expressivity) and integrate various types of data, still maintaining a low burden of computational complexity (Figs. 1 and $2-$ see Methods for the algorithmic details).

In TRalT we estimate the statistical association between a set of genomic events (i.e., mutations, copy number, etc.) annotated in sequencing data by combining optimal graph-based algorithms with bootstrap, hypothesis testing and information theory (Fig. 2). TRalT can reconstruct trees and forests - in general, mutational graphs - which in specific cases can include confluences, to account for the uncertainty on the precedence relation among certain events. Forest models (i.e., disconnected trees), in particular, can stem for possible polyclonal tumour initiation (i.e., tumours with multiple cells of origin [39]), or the presence of tumour-triggering events that are not annotated in the input data (e.g., epigenetic events) (Fig. 1d).

Inputs data in TRalT is represent as binary vectors, which is the standard representation for SCS sequencing and is hereby used to define a unique framework for both multi-region bulk and SCS data (Fig. 1a-c). For a set of cells or regions sequenced, the input reports the presence/absence of $n$ genomic events, for which TRalT will layout a temporal ordering. A binary representation allows to include several types of somatic lesions in the analysis, such as somatic mutations (e.g., singlenucleotide, indels, etc.), copy number alterations, epigenetic states (e.g., methylations, chromatin modifications), etc. (see the Conclusions for a discussion on the issue of data resolution).

\section{Performance evaluation with synthetic simulations}

We assessed the performance of TRalT with both SCS and multi-region data simulated from different types of generative models.

Synthetic data generation. Synthetic single-cell datasets were sampled from a large number of randomly generated topologies (trees or forests) to reflect TRalT's generative model. For each generative topology, binary datasets were generated starting from the root, with a recursive procedure which we describe for the simpler case of a tree: $(i)$ for the root node $x$, the corresponding variable is assigned 1 with a randomly sampled probability $p(x=1)=r$, 
with $r \sim U[0,1]$; (ii) given a branching node $y$ with children $y_{1}, y_{2}, \ldots, y_{n}$, we sample values for the $n$ variables $y_{1}, y_{2}, \ldots, y_{n}$ so that at most one randomly selected child contains 1 , and the others are all 0 . The recursion proceeds from the root to the leaves, and stops whenever a 0 is sampled or a leaf is reached. Note that we are simulating exclusive branching lineages, as one expects from the accumulation of mutations in single cells under the ISA.

As bulk samples usually include intermixed tumour subpopulations, we simulated bulk datasets by pooling singlecell genotypes generated as described above, and setting simulated variables (i.e., mutations) to 1 (= present) in each bulk sample if they appear in the sampled singlecell genotypes more than a certain threshold. More details on these procedures are reported in Section 2 of the Additional file 1.

Consistently with previous studies, we also introduced noise in the true genotypes via inflated false positives and false negatives, which are assumed to have highly asymmetric rates for SCS data. For SCS data we also included missing data in a proportion of the simulated variables [11]. Notice that TRalT can be provided with input noise rates, prior to the inference: therefore, in each reconstruction experiment we provided the algorithm with the noise rates used to generate the datasets, even though mild variations in such input values appear not to affect the inference accuracy - as shown in the noise robustness test presented below and in Fig. 3d.

With a total of $\sim 140.000$ distinct simulations, we could reliably estimate the ability to infer true edges (sensitivity) and discriminate false ones (specificity); further details on parameter settings are available in Section 6 of the Additional file 1. In particular, we compared TRalT's algorithms to SCITE, the state-of-the-art to infer mutational trees from SCS data [11]. We could not include OncoNEM [7] - the benchmark tool for clonal deconvolution - in the comparison, as its computational performance did not scale well with our large number of tests.

In the Main Text we show results for the Edmonds and Chow-Liu algorithms, included in TRalT, and SCITE, in a

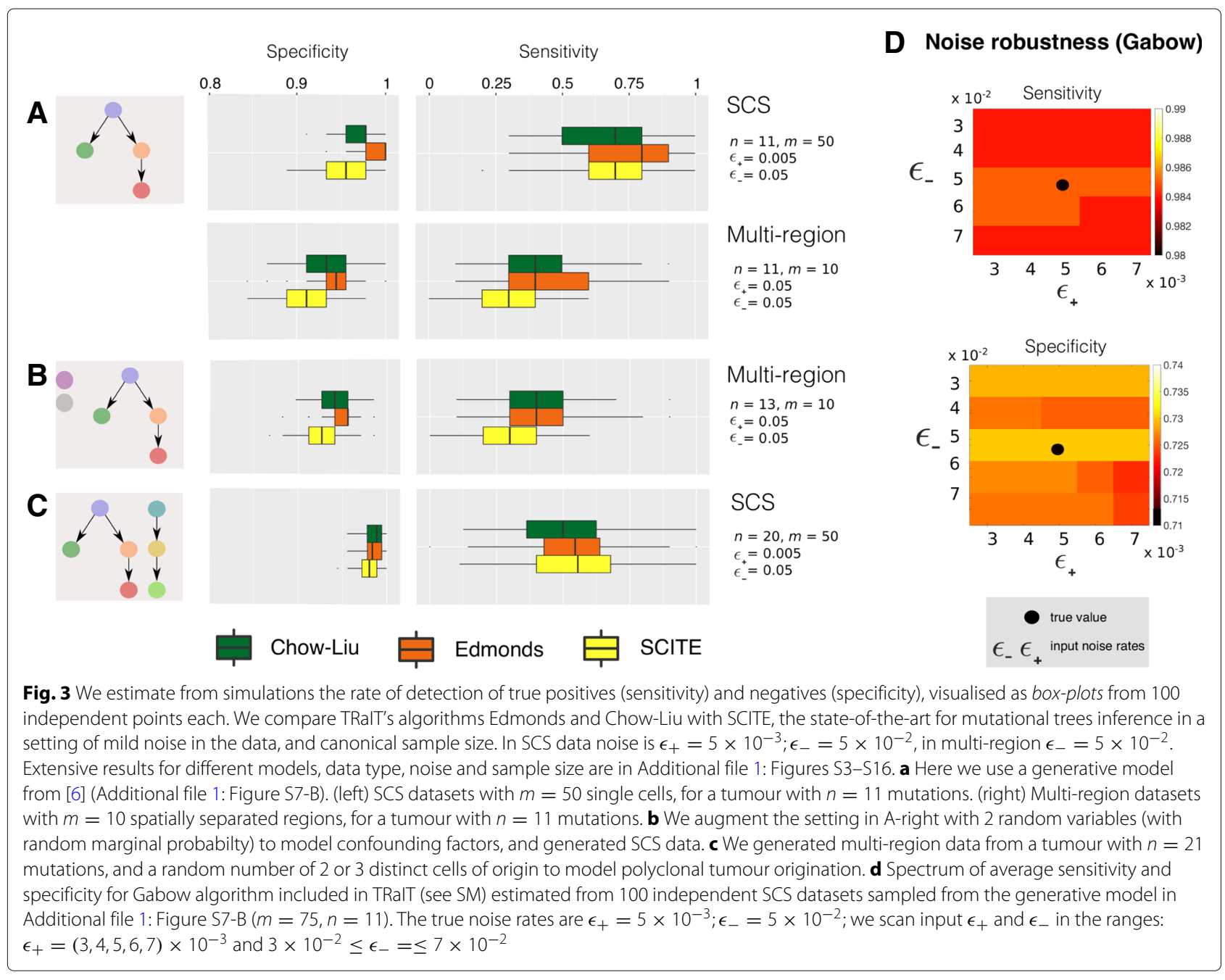


selected number of relevant experimental scenarios. To improve readability of the manuscript, we leave to the Additional file a comprehensive presentation of the results for Gabow, Prim and other approaches [13, 14].

Results from scenario (i), branching evolution. To simulate branching evolution [19], we generated a large number of independent datasets from single-rooted tree structures. In particular, we employed three control polyclonal topologies taken from [6] (Additional file 1: Figure 7) and 100 randomly generated topologies, with a variable number of nodes (i.e., alterations) in the range $n \in[5 ; 20]$. Such generative models were first used to sample datasets with different number of sequenced cells $(m=10,50,100)$. In addition to the noise-free setting, we perturbed data by introducing plausible and highly asymmetric noise rates (i.e., $\epsilon_{+}=\epsilon_{-}=0$ (noise-free); $\epsilon_{+}=0.005, \epsilon_{-}=0.05$; $\epsilon_{+}=0.02, \epsilon_{-}=0.2$.). The same generative topologies were then used to sample multi-region datasets with different number of regions $(m=5,10,20)$, and symmetric noise rates $\left(\epsilon_{+}=\epsilon_{-}=0,0.05,0.2\right)$.

In Fig. 3a we show two selected experimental settings, which are characteristic of the general trends observed on all tests. In particular, one can notice that all the techniques achieve high sensitivity and specificity with SCS data, and significantly lower scores with multi-region data from the same topology; Edmonds displays in general the best results with SCS data (medians $\sim 0.8$ and $\sim 1$ ).

From the results in all simulation settings (Additional file 1: Figures 8 and 9 for the SCS case; Additional file 1: Figures 13 and 14 for the multi-region case), we observe that the overall performance significantly improves for lower noise levels and larger datasets across for all the algorithms, a general result that is confirmed in the other experimental scenarios. In particular, with SCS data, Edmonds and SCITE display similar sensitivity, even though the latter presents (on average) lower specificity, which might point to a mild-tendency to overfit. Results on multi-region data display similar trends, with Edmonds showing the overall best performance and SCITE showing slightly lower performance, especially with small datasets and/or low noise levels. We also specify that, as TRalT's algorithms share the same constraints in the search space and several algorithmic properties, the reduced variance observed across settings is expected.

Results from scenario (ii), confounding factors. To investigate the impact of possible confounding factors on inference accuracy, we introduced in the datasets from scenario $(i)$ a number of random binary variables totally unrelated to the progression. More in detail, we inserted around $n \times 10 \%$ additional random columns in all datasets with $n$ input variables; each additional column is a repeated sampling of a biased coin, with bias uniformly sampled among the marginals of all events.
The performance of TRaIT and SCITE in a selected setting for the multi-region case is shown in Fig. 3b. Surprisingly, the introduction of confounding factors does not impact the performance significantly. In fact, despite two extra variables annotated in the data that are unrelated to the progression, most algorithms still discriminate the true generative model. Similar results are achieved in the SCS case (Additional file 1: Figure 10).

Results from scenario (iii), forest models. Forest topologies can be employed as generative models of tumours initiated by multiple cells, or of tumours whose initiation is triggered by events that are not annotated in the input data. In this test we randomly generated forests with a variable number of distinct disconnected trees, thus assuming that no mutations are shared across the trees. In detail, we generated 100 random forest topologies, with $n=20$ nodes and $q<5$ distinct roots (i.e., disconnected trees), both in the SCS and the multi-region case.

The performance of the tested algorithms in a selected experimental scenario with SCS is shown in Fig. 3c. All algorithms display a clear decrease in sensitivity, with respect to the single-rooted case with similar values of noise and sample size. In the SCS case the performance remarkably increases with larger datasets (median values $\sim 0.75$ with $m=100$ samples in the noise-free case; Additional file 1: Figure 11). Edmonds shows the best tradeoff between sensitivity and specificity, whereas SCITE confirms a mild tendency to overfit for small datasets, yet being very robust against noise. Results from multiregion analysis show an overall decrease in performance (Additional file 1: Figure 16).

Robustness to variations in noise input values. Similarly to other tools, e.g., [7, 11], our algorithms can receive rates of false positives and negatives in the data $\left(\epsilon_{+}\right.$and $\left.\epsilon_{-}\right)$as input. Thus, we analyzed the effect of miscalled rates on the overall performance. More in detail, we analyzed the variation of the performance of Gabow and SCITE, on a dataset generated from a generative tree with intermediate complexity ("Medium" topology in Additional file 1: Figure 7), with $n=11$ nodes and $m=75$ samples, $\epsilon_{+}=5 \times 10^{-} 3$ and $\epsilon_{-}=5 \times 10^{-} 2$. We scanned 25 possible combinations of input $\epsilon_{+}$and $\epsilon_{-}$in the following ranges: $\epsilon_{+}=(3,4,5,6,7) \times 10^{-3}$ and $\epsilon_{-}=$ $(3,4,5,6,7) \times 10^{-2}$. Results in Fig. 3d and Additional file 1: Tables 4 and 5 show no significant variations of the performance with different combinations of input values for $\epsilon_{+}$ and $\epsilon_{-}$, for both algorithms. This evidence also supports our algorithmic design choice which avoids sophisticate noise-learning strategies in TRaIT, a further reason that speeds up computations.

Missing data. Significant rates of missing data are still quite common in SCS datasets, mainly due to amplification biases during library preparation. We evaluated the impact of missing data by using 20 benchmark single-cell 
datasets which were generated from a tree with $n=11$ nodes (Additional file 1: Figure 7). For every dataset we simulated the calling of mutations from $m=75$ single sequenced cells, and in half of the cases (i.e., 10 datasets) we also imputed extra error rates in the data to model sequencing errors. In particular, we introduced false positives and false negative calls with rates $\epsilon_{+}=0.005$ and $\epsilon_{-}=0.05$. On top of this, for each of the 20 datasets we generated 5 configurations of missing data (uniformly distributed), using as measure the percentage $r$ of missing data over the total number of observations. A total of 100 distinct datasets have been obtained using $r=$ 0, 0.1, 0.2, 0.3, 0.4 (i.e., up to $40 \%$ missing data). As SCITE can explicitly learn parameters from missing data, we run the tool with no further parameters. Instead, for TRaIT's algorithms, we performed the following procedure: for each dataset $D$ with missing data, we imputed the missing entries via a standard Expectation-Maximization (EM) algorithm, repeating the procedure to generate $100 \mathrm{com}$ plete datasets $\left(D_{1}, \ldots, D_{100}\right)$. To asses the performance of each algorithm, we computed the fit to all the 100 datasets, and selected the solution that maximised the likelihood of the model.

We present in Fig. 4 the results of this analysis for Edmonds and Chow-Liu algorithms included in TRalT, and for SCITE; results for Gabow and Prim algorithms are presented in Additional file 1: Figure 12. In general, missing data profoundly affect the performance of all methods. SCITE shows overall more robust sensitivity, in spite of slightly worse specificity. The performance is always significantly improved when data do not harbour noise and, in general, is reasonably robust up to $30 \%$ missing data.

Computational time. One of the major computational advantages of TRalT is its scalability, which will be essential in anticipation of the increasingly larger SCS datasets expected in the near future. In this respect, we have observed across all tests a $3 \times$ speedup of TRalT's algorithms on standard CPUs with respect to SCITE, and a $40 \times$ speedup with respect to OncoNEM (Additional file 1: Table 6).

\section{Analysis of patient-derived multi-region data for a MSI-high colorectal cancer}

We applied TRalT to 47 nonsynonymous point mutations and 11 indels detected via targeted sequencing in patient P3 of [40]. This patient has been diagnosed with a moderately-differentiated MSI-high colorectal cancer, for which 3 samples are collected from the primary tumour (P3-1, P3-2, and P3-3) and two from a right hepatic lobe metastasis L-1 and L-2 (Fig. 5a). To prepare the data for our analyses, we first grouped mutations occurring in the same regions. We obtained: $(a)$ a clonal group of 34 mutations detected in all samples $(b)$ a subclonal group of 3 mutations private to the metastatic regions, and $(c)$
8 mutations with distinct mutational profiles. The clonal group contains mutations in key colorectal driver genes such as APC, KRAS, PIK3CA and TP53 [15],

Edmonds's model predicts branching evolution and high levels of ITH among the subclonal populations, consistently with the original phylogenetic analysis by $\mathrm{Lu}$ et al. [40] (Fig. 5b). In particular, the subclonal trajectory that characterizes the primary regions is initiated by a stopgain SNV in the DNA damage repair gene ATM, whereas the subclonal metastatic expansion seems to originate by a stopgain SNV in GNAQ, a gene reponsible for diffusion in many tumour types [41]. The model also pictures two distinct trajectories with different mutations in SMAD4: a nonsynonimous SNV in group L, and a stopgain $\mathrm{SNV}$ in two regions of the primary. Interestingly, SMAD4 regulates cell proliferation, differentiation and apoptosis [42], and its loss is correlated with colorectal metastases [43].

We applied SCITE to the same data (Additional file 1: Figure S22), and compared it to Edmonds. Both models depict the same history for the metastatic branch, but different tumour initiation: SCITE places the ATM mutation on top of the clonal mutations, which appear ordered in a linear chain of 34 events. However, this ordering is uncertain because SCITE's posterior is multi-modal (i.e., several orderings have the same likelihood; Additional file 1: Figure 22). Further comments on the results, and outputs from other algorithms are available Supplementary Material (Additional file 1: Figure 21).

\section{Analysis of patient-derived SCS data for a triple-negative breast cancer}

We applied TRaIT to the triple-negative breast cancer patient TNBC of [34]. The input data consists of singlenucleus exome sequencing of 32 cells: 8 aneuploid (A) cells, 8 hypodiploid $(\mathrm{H})$ cells and 16 normal cells $(\mathrm{N})$ (Fig. 6a). Wang et al considered clonal all mutations detected in a control bulk sample and in the majority of the single cells, and as subclonal those undetected in the bulk [34]; all mutations were then used to manually curate a phylogenetic tree (Fig. 6b).

We run TRaIT on all single cells, with nonsynonymous point mutations annotated in 22 genes, and set $\epsilon_{+}=$ $1.24 \times 10^{-6}$ and $\epsilon_{-}=9.73 \times 10^{-2}$ as suggested in [34]. All TRalT's algorithms return tree topologies (Additional file 1: Figures 17-18); Fig. 6c shows the model obtained with Edmonds. We integrate the analysis by applying SCITE to the same data, and by computing prevalence and evolutionary relations of putative clones with OncoNEM as well (Fig. 6d).

TRalT provides a finer resolution to the original analysis by Wang et al. [34], and retrieves gradual accumulation of point mutations thorough tumour evolution, which highlight progressive DNA repair and replication 


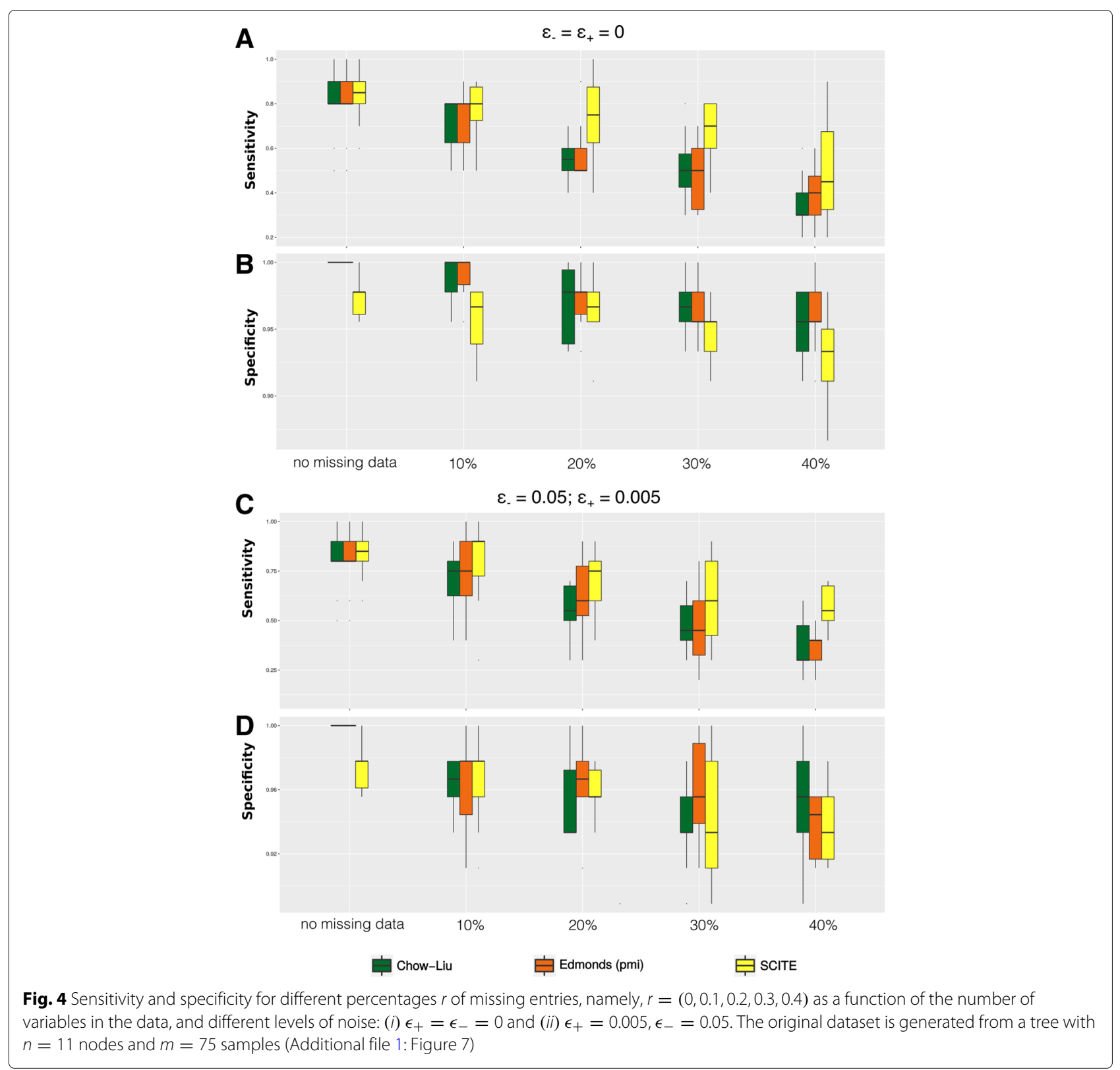

deregulation. The model also predicts high-confidence branching evolution patterns consistent with subclones $A_{1}$ (PPP2R1A, SYNE2 and AURKA), $A_{2}$ (ECM2, CHRM5 and TGFB2), and $\mathrm{H}$ (NRRK1, AFF4, ECM1, CBX4), and provides an explicit ordering among clonal mutations in PTEN, TBX3 and NOTCH2, which trigger tumour initiation. Interestingly, TRaIT also allows to formulate new hypotheses about a possibly undetected subclone with private mutations in JAK1, SETBP1 and CDH6. Finally, we note that that temporal ordering among mutations in ARAF, AKAP9, NOTCH3 and JAK1 cannot be retrieved, since these events have the same marginal probability in these data.
By applying SCITE to these data with the same noise rates, we retrieved 10.000 equivalently optimal trees. The overlap between the first of the returned trees (Additional file 1: Figure S19) and ours is poor (8 out of 19 edges), and SCITE's models contain a long linear chain of 13 truncal mutations. Clonal deconvolution analysis via OncoNEM allowed us to detect 10 clones, their lineages and evolutionary relations. This analysis is in stronger agreement with ours, and the estimated mutational ordering obtained by assigning mutations to clones (via maximum a posteriori, as suggested in [7]) largely overlaps with TRalT's predictions. This is particularly evident for early events, and for most of the late subclonal ones, exception 
A Colorectal cancer Multi-region P3 (Lu et al.)

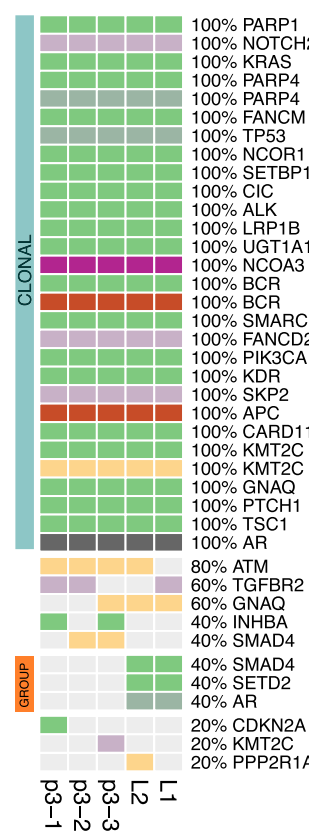

Mutations

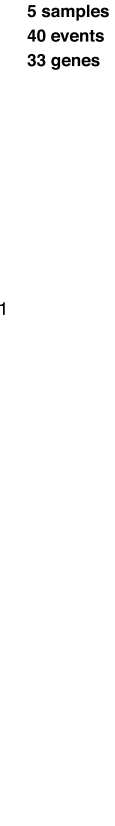

\section{B TRalT's model (Edmonds)}

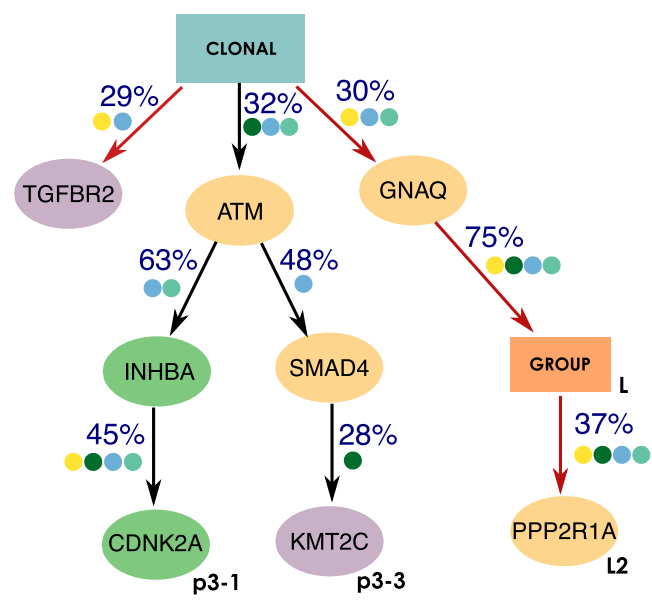

$\longrightarrow p<0.05$ Temporal priority, Probability raising, Hypergeometric test

$p>0.05$ Hypergeometric test

$\%$ Non-parametric Bootstrap

Retrieved by SCITE

Retrieved by Chow-Liu

Retrieved by Gabow

Retrieved by Prim

made for subclone $\mathrm{H}$, which is not detected by OncoNEM. These results prove that concerted application of tools for mutational and clonal trees inference can provide a picture of ITH at an unprecedented resolution.

\section{Discussion}

In this paper we have introduced TRalT, a computational approach for the inference of cancer evolution models in single tumours. TRalT's expressive framework allows to reconstruct models beyond standard trees, such as forests, which capture different modalities of tumour initiation (e.g., by multiple cells of origin, or by events missing in available genomic data, such as epigenetic states) and, under certain conditions of data and parameters, confluences. Future works will exploit this latter feature to define a comprehensive modelling framework that accounts for explicit violations of the ISA, in order to model further evolutionary phenomena, such as convergent (parallel) evolution and back mutations [37].
TRalT is based on a binary representation of input data, for both multi-region and single-cell sequencing data. We comment on this design choice concerning the case of multi-region bulk data, because most methods that process bulk data use allelic frequencies and cancer cell fractions to deconvolve the clonal composition of a tumor (see, e.g., [29, 30, 44]). In this respect, allele frequencyderived inputs provide higher-resolution estimates of the temporal orderings among samples. In fact, if two mutations co-occur in the same set of samples, their relative temporal ordering cannot be determined from a binary input, while this might be possible from their cancer cell fractions. However, despite the lower resolution, a binary representation is still a viable option in multi-region analyses.

First, binary data can describe the presence or absence of a wide range of covariates, which otherwise might be difficult or impossible to represent with allelefrequencies or cancer cell fractions. These include, 
A Breast cancer

Single nucleus genome sequencing, Patient TNBC (Wang et al.)

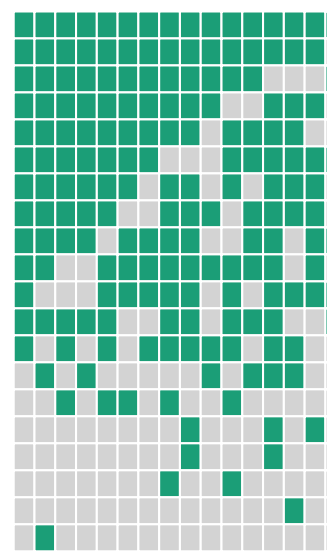

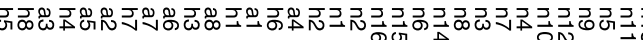

\section{TRalT's model (Edmonds)}

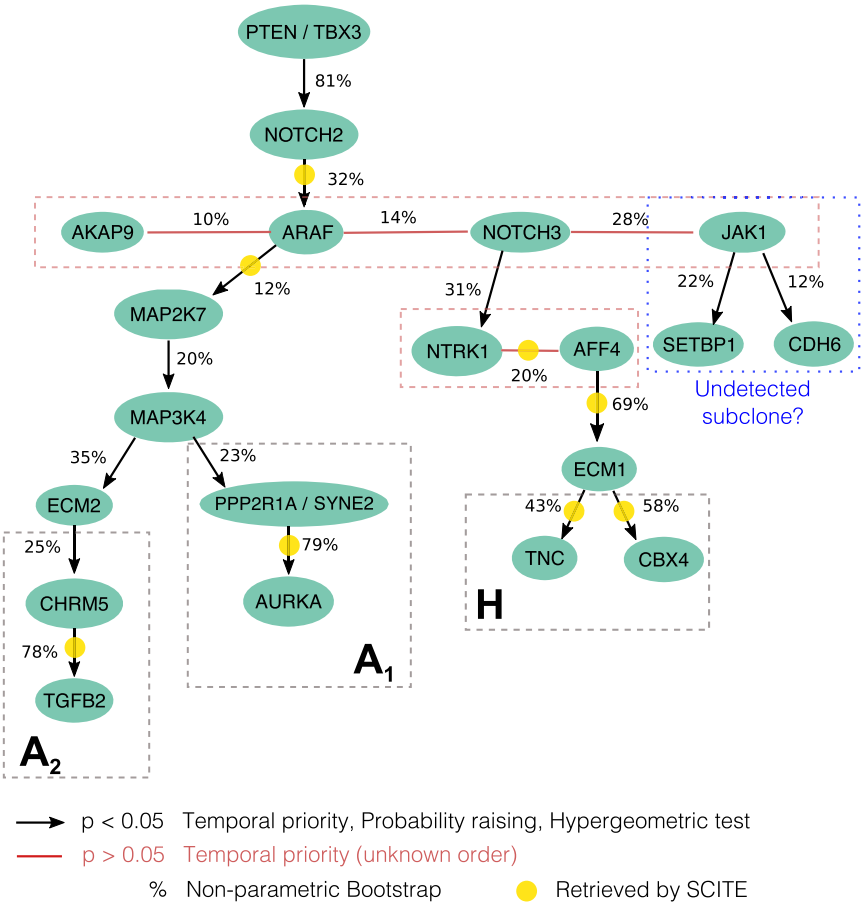

\section{B Phylogeny}

Manual curation (Wang et al.)

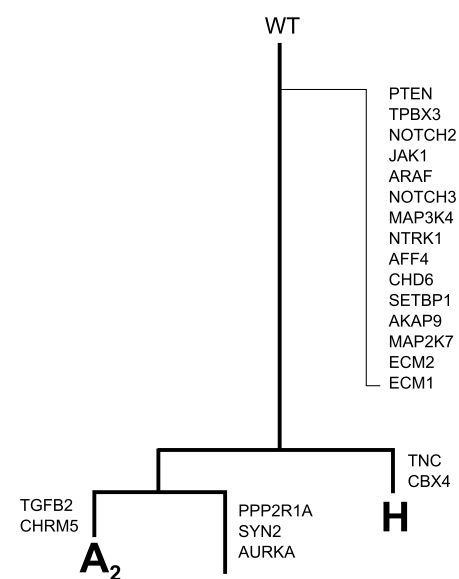

$\mathrm{A}_{1}$

\section{Clonal decomposition (OncoNEM)}

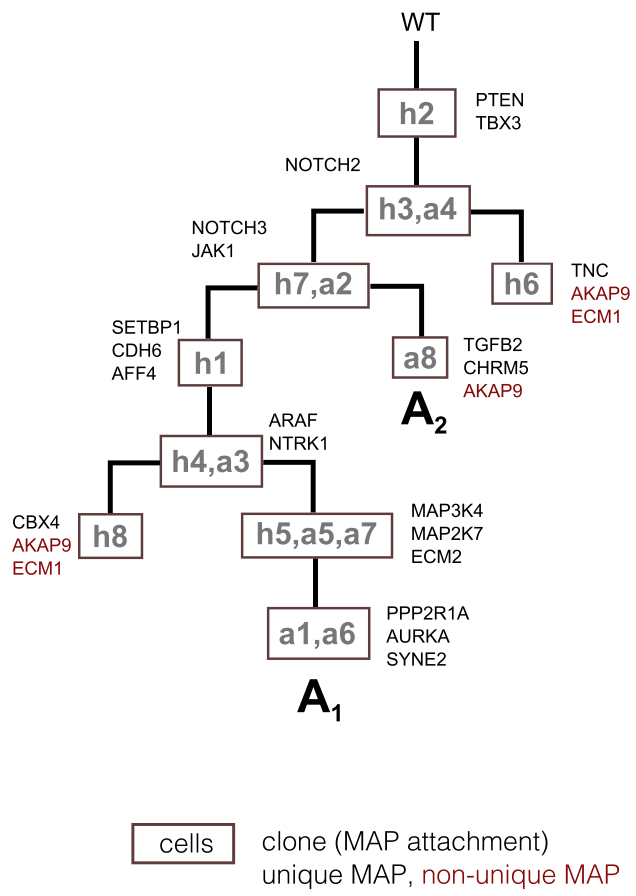

Fig. 6 a Input data from single-nucleus sequencing of 32 cells from a triple-negative breast cancer [34]. As the rate of missing values in the original data was around 1\%, the authors set all missing data points equal to 0 ; in the dataset, allelic dropout is equal to $9.73 \times 10^{-2}$, and false discovery equal to $1.24 \times 10^{-6}$. b Phylogenetic tree manually curated in [34]. Mutations are annotated to the trunk if they are ubiquitous across cells and a bulk control sample. Subclonal mutations appearing only in more than one cell. c. Mutational graph obtained with Edmonds algorithm; p-values are obtained by 3 tests for conditions (Eq. 1) and overlap (hypergeometric test), and edges annotated with a posteriori non-parametric bootstrap scores (100 estimates). For these data, all TRalT's algorithms return trees (Additional file 1: Figure S17-18), consistently with the manually curated phylogeny (A). Most edges are highly confident ( $p<0.05$ ), except for groups of variables with the same frequency which have unknown ordering (red edges). The ordering of mutations in subclones $\mathrm{A}_{1}, \mathrm{~A}_{2}$ and tumour initiation has high bootstrap estimates (> 75\%). Yellow circles mark the edges retrieved also by SCITE. $\mathbf{d}$. We also performed clonal tree inference with OncoNEM, which predicts 10 clones. Mutations are assigned to clones via maximum a posteriori estimates. The mutational orderings of the early clonal expansion of the tumour and of most of the late subclonal events are consistent with TRalT's prediction 
for instance, complex structural re-arrangements, structural variants, epigenetic modifications, over/under gene expression states and high-level pathway information. The integration of such heterogeneous data types and measurements will be essential to deliver an effective multi-level representation of the life history of individual tumours. Methods that strictly rely on allelic frequencies might need to be extended to accommodate such data types.

Second, binary inputs can be used to promptly analyse targeted sequencing panels, whereas the estimation of subclonal clusters from allele frequencies (i.e., via subclonal deconvolution) requires at least high-depth wholeexome sequencing data to produce reliable results. While it is true that whole-exome and whole-genome assays are becoming increasingly common, many large-scale genomic studies are still relying on targeted sequencing (see, e.g., $[45,46]$ ), especially in the clinical setting. A prominent example are assays for longitudinal sampling of circulating tumour DNA during therapy monitoring, which often consist of deep-sequencing target panels derived from the composition of a primary tumour (see, e.g., [47]).

Finally, binary inputs can be obtained for both bulk and single-cell sequencing data, and this in turn allows to use the same framework to study cancer evolution from both data types. This is innovative, and in the future integrative methods might draw inspiration from our approach.

\section{Conclusions}

Intra-tumour heterogeneity is a product of the interplay arising from competition, selection and neutral evolution of cancer subpopulations, and is one of the major causes of drug resistance, therapy failure and relapse [48-52]. For this reason, the choice of the appropriate statistical approach to take full advantage of the increasing resolution of genomic data is key to produce predictive models of tumour evolution with translational relevance.

We have here introduced TRalT, a framework for the efficient reconstruction of single tumour evolution from multiple-sample sequencing data. Thanks to the simplicity of the underlying theoretical framework, TRalT displays significant advancements in terms of robustness, expressivity, data integration and computational complexity. TRalT can process both multi-region and SCS data (separately), and its optimal algorithms maintain a low computational burden compare to alternative tools. TRalT's assumptions to model accumulation phenomena lead to accurate and robust estimate of temporal orderings, also in presence of noisy data.

We position TRalT in a very precise niche in the landscape of tools for cancer evolution reconstruction, i.e., that of methods for the inference of mutational trees/graphs (not clonal or phylogenetic trees), from binary data (alteration present/absent), and supporting both multi-region bulk and single-cell sequencing data. We advocate the use of TRalT as complementary to tools for clonal tree inference, in a joint effort to quantify the extent of ITH, as shown in the case study on triple negative breast cancer.

\section{Methods}

\section{Input Data and Data Types}

TRalT processes an input binary matrix $D$ with $n$ columns and $m$ rows. $D$ stores $n$ binary variables (somatic mutations, CNAs, epigenetic states, etc.) detected across $m$ samples (single cells or multi-region samples) (Fig. 2a). One can annotate data at different resolutions: for instance, one can distinguish mutations by type (missense vs truncating), position, or context ( $\mathrm{G}>\mathrm{T}$ vs $\mathrm{G}>\mathrm{A}$ ), or can just annotate a general "mutation" status. The same applies for copy numbers, which can be annotated at the focal, cytoband or arm-level. In general, if an entry in $D$ is 1 , then the associated variable is detected in the sample.

In our framework we cannot disentangle the temporal ordering between events that occur in the same set of samples. These will be grouped by TRalT in a new "aggregate" node, prior to the inference (Fig. 2b). TRalT does not explicitly account for back mutations due to loss of heterozygosity. Yet, the information on these events can be used to prepare input data if one matches the copy number state to the presence of mutations. By merging these events we can retrieve their temporal position in the output graph (Additional file 1: Figure S23).

TRalT supports both multi-region and SCS data. As we expect $D$ to contain noisy observations of the unknown true genotypes, the algorithms can be informed of false positives and negatives rates $\left(\epsilon_{+} \geq 0\right.$ and $\left.\epsilon_{-} \geq 0\right)$. TRalT does not implement noise learning strategies, similarly to OncoNEM [11]. This choice is sensitive if the algorithms show stable performance for slight variations in the input noise rates, especially when reasonable estimates of $\epsilon_{+}$and $\epsilon_{-}$can be known a priori. This feature allows TRalT to be computationally more efficient, as it avoids to include a noise learning routine in the fit. Missing data, instead, are handled by a standard Expectation Maximisation approach to impute missing values: for every complete dataset obtained, the fit is repeated and the model that maximises the likelihood across all runs is returned.

\section{TRalT's Procedure}

All TRalT's algorithms can be summarised with a threesteps skeleton, where the first two steps are the same across all algorithms. Each algorithm will return a unique output model, whose post hoc confidence can be assessed via cross-validation and bootstrap [15]. 


\section{Step 1: assessment of statistical association - Fig. $2 c$}

We estimate the statistical association between events by assessing two conditions inspired to Suppes' theory of probabilistic causation, which is particularly sound in modelling cumulative phenomena [38].

Let $p(\cdot)$ be an empirical probability (marginal, joint, conditional etc.) estimated from dataset $D$. Conditions on (i) temporal direction and (ii) association's strength are assessed as follows: for every pair of variables $x$ and $y$ in $D$, $x$ is a plausible temporally antecedent event of $y$ if

$$
p(x)>p(y) \wedge p(y \mid x)>p(y \mid \neg x) .
$$

The former condition acts as the Infinite Sites Assumption (ISA), as we assume that alterations are inherited across cell divisions (i.e., somatic): thus, the comparison of marginal frequencies is a proxy to compute the relative ordering among events. The latter condition, instead, implies statistical dependence: $p(x, y)>$ $p(x) p(y)[13]$.

Both conditions are assessed among all variables pairs via non-parametric bootstrap and a one-tailed MannWhitney test: only if both conditions are statistically significant at some $\alpha$-level (e.g., 0.05), the edge connecting the variable pair will be included in a primafacie direct graph $G_{\mathrm{pf}}$. Edges in $G_{\mathrm{pf}}$ are candidate to be selected in the final output model, and thus we are reducing the search space via the the above conditions, which are necessary but not sufficient. These conditions have been previously used to define causal approaches for cancer progression [14, 15]; see further discussion in Supplementary Material. This step has asymptotic complexity $\mathcal{O}\left((n m)^{2} \times B\right)$ where $B$ is the cost of bootstrap and hypothesis testing on each entry in $D$. Notice that this procedure can create disconnected components.

\section{Step 2: loop removal - Fig. $2 d$}

GPF can contain loops, which we have to remove to model an accumulation process. Loops may arise when an arc between a pair of nodes cannot be unequivocally directed, e.g., due to small sample size which leads to uncertain bootstrap estimations. TRalT renders acyclic $G_{\mathrm{PF}}$ by using heuristic strategies that remove less confident edges (see [14]); the output produced is a new graph $G_{\mathrm{NL}}$.

\section{Step 3: reconstruction of the output model - Fig. $2 e-f$}

We render $G_{\mathrm{NL}}$ a weighted graph by annotating its edges via information-theoretic measures such as point-wise mutual information and the like. Then, we can exploit 4 different off-the-shelf algorithms to reconstruct an output model $G_{\mathrm{MO}}$ from $G_{\mathrm{NL}}$. $G_{\mathrm{MO}}$ will be either a tree or a forest with multiple roots, and the complexity of this step depends on the adopted algorithm. Notably, all algorithms currently incorporated in TRaIT have theoretically-optimal worst-case polynomial complexity. We describe two of them (Edmonds and Chow-Liu), and leave the description of the other techniques (Gabow and Prim) to the Supplementary Material.

- Edmonds is an algorithm for the inference of weighted directed minimum spanning trees [53]: it scan $G_{\mathrm{NL}}$ to identify the tree that maximises the edges' weights. Spanning trees have been previously applied to cancer $[54,55]$. Yet, TRalT is the only framework to constraint spanning trees by condition (1);

- Chow-Liu's algorithm is a method to compute a factorisation of a joint distribution over the input variables [56]. Chow-Liu reconstructs undirected trees by definition; we assign the direction to each edge so that the event with higher marginal probability is on top, mirroring condition (1). Confluences in $G_{\mathrm{MO}}$ can emerge under certain conditions of the observed probabilities, which account for the uncertainty on the temporal precedence among events (technically, in such cases we reconstruct direct acyclic graphs, DAGs - see the Supplementary Material for details).

In all TRalT's algorithms, if $G_{\mathrm{NL}}$ includes $k$ disconnected components, then the output model $G_{\mathrm{MO}}$ will include $k$ disconnected trees.

In term of complexity, we note that all TRalT's algorithms are optimal polynomial-time algorithmic solutions to each of their corresponding combinatorial problems. Thus, they scale well with sample size, a problem sometimes observed with Bayesian approaches that cannot compute a full posterior on the model parameters. Quantitative assessment of TRalT's scalability with large datasets is provided as Supplementary Material (Additional file 1: Table 7), where we show that many thousands of cells can be processed in a few seconds.

\section{Tumour evolution scenarios}

TRalT can infer mutational graphs in the following scenarios (see Fig. 1d):

1. Branching evolution (including linear evolution as subcase): in this case TRalT will return a tree with one root and zero disconnected components.

2. Presence of confounding factors in $D$ (e.g., miscalled mutations): TRalT will reconstruct a model with disconnected individual nodes.

3. Polyclonal origin due to multiple cells of tumour origin, or to upstream events triggering tumour development that missing in $D$ (e.g., epigenetic events): TRalT will return models with disconnected components (i.e., forests).

In general, we recommend to apply all TRalT's algorithms and to compare the output models; the creation 
of a consensus model is an option to rank the edges detected across several methods, as we show in the case studies.

\section{Additional file}

Additional file 1: Additional file 1: Data.Extensive description of all the methods and experiments ran with TRaIT, both on simulated data and on real data. In-depth description of the simulated data generation algorithms and table summaries of $\sim 140.00$ simulations. (PDF $4507 \mathrm{~kb}$ )

\section{Abbreviations}

CNA: Copy-Number Alteration; CT: Clonal Tree; ISA: Infinite Sites Assumption; ITH: Intra-Tumour Heterogeneity; MSI: Micro-Satellite Instable; SCS: Single-Cell Sequencing; SNV: Single-Nucleotide Variant

\section{Acknowledgements}

We thank Bud Mishra for valuable insights on the effects of parallel evolution to our framework. We also thank Guido Sanguinetti and Yuanhua Huang for useful discussions on the preliminary version of this manuscript.

\section{Funding}

This work was partially supported by the Elixir Italian Chapter and the SysBioNe project, a Ministero dell'Istruzione, dell'Universitá e della Ricerca initiative for the Italian Roadmap of European Strategy Forum on Research Infrastructures. The funding body did not play any role in the design of the study and collection, analysis, and interpretation of data and in writing the manuscript.

\section{Availability of data and materials}

TRalT is included in the TRONCO R suite for TRanslational ONCOlogy, available at its webpage http://troncopackage.org and mirrored at Bioconductor. All data used in this paper are available from the supplementary material of [34] and [40]. We provide the source code and the input data to reproduce the case studies at: BIMIB-DISCo/TRaIT.

\section{Authors' contributions}

$D R, A G$ and GC designed the algorithmic framework. DR, LDS and GC implemented the tool. LDS carried out the simulations on synthetic data. Data gathering was performed by DR, AG, LDS and GC. All the authors analyzed the results and interpreted the models. DR, AG, MA and GC wrote the draft of the paper, which all authors reviewed and revised.

\section{Ethics approval and consent to participate}

Not applicable.

\section{Consent for publication}

Not applicable.

\section{Competing interests}

The authors declare that they have no competing interests.

\section{Publisher's Note}

Springer Nature remains neutral with regard to jurisdictional claims in published maps and institutional affiliations.

\section{Author details \\ ${ }^{1}$ Department of Pathology, Stanford University, 94305 Palo Alto, CA, USA ${ }^{2}$ Dipartimento di Informatica, Sistemistica e Comunicazione, Università degli Studi di Milano-Bicocca, Viale Sarca 336, 20126 Milan, Italy. ${ }^{3}$ Institute of Molecular Bioimaging and Physiology of the Italian National Research Council (IBFM-CNR), Viale F.lli Cervi 93, 20090 Segrate, Milan, Italy. ${ }^{4}$ Milan Center for Neuroscience, Università degli Studi di Milano-Bicocca, San Gerardo Hospital, Via Pergolesi 33, 20052 Monza, Italy. ${ }^{5}$ Centre for Evolution and Cancer, The Institute of Cancer Research, 15 Cotswold Road, SM2 5NG London, UK.}

\section{References}

1. Beerenwinkel N, Schwarz RF, Gerstung M, Markowetz F. Cancer evolution: mathematical models and computational inference. Syst Biol. 2015:64(1):1-25.

2. Schwartz R, Schäffer AA. The evolution of tumour phylogenetics: principles and practice. Nat Rev Genet. 2017;18(4):213.

3. Caravagna G, Giarratano Y, Ramazzotti D, Tomlinson I, Graham TA, Sanguinetti G, Sottoriva A. Detecting repeated cancer evolution from multi-region tumor sequencing data. Nat Methods. 2018;15(9):707-14. https://doi.org/10.1038/s41592-018-0108-X.

4. Davis A, Navin NE. Computing tumor trees from single cells. Genome Biol. 2016;17(1):113.

5. Stamatakis A. Raxml version 8: a tool for phylogenetic analysis and post-analysis of large phylogenies. Bioinformatics. 2014;30(9):1312-1313.

6. Yuan K, Sakoparnig T, Markowetz F, Beerenwinkel N. BitPhylogeny: a probabilistic framework for reconstructing intra-tumor phylogenies. Genome Biol. 2015;16(1):1.

7. Ross EM, Markowetz F. OncoNEM: inferring tumor evolution from single-cell sequencing data. Genome Biol. 2016;17(1):1.

8. Roth A, McPherson A, Laks E, Biele J, Yap D, Wan A, Smith MA, Nielsen CB, McAlpine JN, Aparicio S, Bouchard-Côté A, Shah SP. Clonal genotype and population structure inference from single-cell tumor sequencing. Nat Methods. 2016;13(7):573-6.

9. Zafar H, Tzen A, Navin N, Chen K, Nakhleh L. SiFit: inferring tumor trees from single-cell sequencing data under finite-sites models. Genome Biol. 2017;18(1):178.

10. Kim Kl, Simon R. Using single cell sequencing data to model the evolutionary history of a tumor. BMC Bioinforma. 2014;15(1):27.

11. Jahn K, Kuipers J, Beerenwinkel N. Tree inference for single-cell data. Genome Biol. 2016;17(1):1

12. Salehi S, Steif A, Roth A, Aparicio S, Bouchard-Côté A, Shah SP. ddClone: joint statistical inference of clonal populations from single cell and bulk tumour sequencing data. Genome Biology. 2017;18(1):44

13. Loohuis LO, Caravagna G, Graudenzi A, Ramazzotti D, Mauri G, Antoniotti M, Mishra B. Inferring tree causal models of cancer progression with probability raising. PloS ONE. 2014;9(10):108358.

14. Ramazzotti D, Caravagna G, Loohuis LO, Graudenzi A, Korsunsky I, Mauri G, Antoniotti M, Mishra B. CAPRI: efficient inference of cancer progression models from cross-sectional data. Bioinformatics. 2015;31(18):3016-26.

15. Caravagna G, Graudenzi A, Ramazzotti D, Sanz-Pamplona R, De Sano L, Mauri G, Moreno V, Antoniotti M, Mishra B. Algorithmic methods to infer the evolutionary trajectories in cancer progression. Proc Natl Acad Sci. 2016:113(28):4025-34. https://doi.org/10.1073/pnas.1520213113.

16. Ramazzotti D. A model of selective advantage for the efficient inference of cancer clonal evolution. PhD thesis: Università degli Studi di Milano-Bicocca; 2017. arXiv preprint arXiv:1602.07614. https://arxiv.org/ abs/1602.07614

17. Ramazzotti D, Nobile MS, Cazzaniga P, Mauri G, Antoniotti M. Parallel implementation of efficient search schemes for the inference of cancer progression models. In: Computational Intelligence in Bioinformatics and Computational Biology (CIBCB), 2016 IEEE Conference On. IEEE; 2016. p. 1-6.

18. Ramazzotti D, Graudenzi A, Caravagna G, Antoniotti M. Modeling cumulative biological phenomena with suppes-bayes causal networks. Evol Bioinforma. 2018;14:1176934318785167.

19. Gerlinger M, Rowan AJ, Horswell S, Larkin J, Endesfelder D, Gronroos E, Martinez P, Matthews N, Stewart A, Tarpey P, et al. Intratumor heterogeneity and branched evolution revealed by multiregion sequencing. N Engl J Med. 2012;366(10):883-892.

20. de Bruin EC, McGranahan N, Mitter R, Salm M, Wedge DC, Yates L, Jamal-Hanjani M, Shafi S, Murugaesu N, Rowan AJ, et al. Spatial and temporal diversity in genomic instability processes defines lung cancer evolution. Science. 2014;346(6206):251-6.

21. Zhang J, Fujimoto J, Zhang J, Wedge DC, Song X, Zhang J, Seth S, Chow C-W, Cao Y, Gumbs C, et al. Intratumor heterogeneity in localized lung adenocarcinomas delineated by multiregion sequencing. Science. 2014;346(6206):256-9.

22. Yates LR, Gerstung M, Knappskog S, Desmedt C, Gundem G, Van Loo P, Aas T, Alexandrov LB, Larsimont D, Davies H, et al. Subclonal diversification of primary breast cancer revealed by multiregion sequencing. Nat Med. 2015;21(7):751-9. 
23. Jamal-Hanjani M, Wilson GA, McGranahan N, Birkbak NJ, Watkins TB, Veeriah S, Shafi S, Johnson DH, Mitter R, Rosenthal R, et al. Tracking the evolution of non-small-cell lung cancer. N Engl J Med. 2017;376(22): 2109-21.

24. Oesper L, Mahmoody A, Raphael BJ. Inferring intra-tumor heterogeneity from high-throughput dna sequencing data. Lect Notes Comput Sci. 2013;7821:171-2. https://doi.org/10.1007/978-3-642-37195-0_14.

25. Strino F, Parisi F, Micsinai M, Kluger Y. TrAp: a tree approach for fingerprinting subclonal tumor composition. Nucleic Acids Res. 2013;41(17):165.

26. Roth A, Khattra J, Yap D, Wan A, Laks E, Biele J, Ha G, Aparicio S, Bouchard-Côté A, Shah SP. PyClone: statistical inference of clonal population structure in cancer. Nat Methods. 2014;11(4):396-8.

27. Jiao W, Vembu S, Deshwar AG, Stein L, Morris Q. Inferring clonal evolution of tumors from single nucleotide somatic mutations. BMC Bioinforma. 2014;15(1):1.

28. Zare H, Wang J, Hu A, Weber K, Smith J, Nickerson D, Song C, Witten D, Blau CA, Noble WS. Inferring clonal composition from multiple sections of a breast cancer. PLoS Comput Biol. 2014;10(7):1003703.

29. Deshwar AG, Vembu S, Yung CK, Jang GH, Stein L, Morris Q. PhyloWGS: reconstructing subclonal composition and evolution from whole-genome sequencing of tumors. Genome Biol. 2015;16(1):1.

30. El-Kebir M, Satas G, Oesper L, Raphael BJ. Inferring the mutational history of a tumor using multi-state perfect phylogeny mixtures. Cell Syst. 2016:3(1):43-53.

31. Hu Z, Curtis C. Inferring Tumor Phylogenies from Multi-region Sequencing. Cell Syst. 2016;3(1):12-4.

32. Navin N, Kendall J, Troge J, Andrews P, Rodgers L, Mclndoo J, Cook K, Stepansky A, Levy D, Esposito D, et al. Tumour evolution inferred by single-cell sequencing. Nature. 2011;472(7341):90-4.

33. Gawad C, Koh W, Quake SR. Dissecting the clonal origins of childhood acute lymphoblastic leukemia by single-cell genomics. Proc Natl Acad Sci. 2014;111(50):17947-52.

34. Wang $Y$, Waters J, Leung ML, Unruh A, Roh W, Shi $X$, Chen $K$, Scheet $P$, Vattathil S, Liang $\mathrm{H}$, et al. Clonal evolution in breast cancer revealed by single nucleus genome sequencing. Nature. 2014;512(7513):155-60.

35. Navin NE. The first five years of single-cell cancer genomics and beyond Genome Res. 2015;25(10):1499-507.

36. Schwarz RF, Trinh A, Sipos B, Brenton JD, Goldman N, Markowetz F. Phylogenetic quantification of intra-tumour heterogeneity. PPLoS Comput Biol. 2014;10(4):1003535.

37. Navin NE, Chen K. Genotyping tumor clones from single-cell data. Nat Methods. 2016;13(7):555-6.

38. Suppes P. A Probabilistic Theory of Causality. The Netherlands: North-Holland Publishing Company Amsterdam; 1970.

39. Parsons BL. Many different tumor types have polyclonal tumor origin: evidence and implications. Mutat ResRev Mutat Res. 2008;659(3):232-47.

40. Lu Y-W, Zhang H-F, Liang R, Xie Z-R, Luo H-Y, Zeng Y-J, Xu Y, Wang L-M, Kong $X-Y$, Wang K-H. Colorectal cancer genetic heterogeneity delineated by multi-region sequencing. PloS ONE. 2016;11(3):0152673.

41. Kim C-Y, Kim DW, Kim K, Curry J, Torres-Cabala C, Patel S. GNAQ mutation in a patient with metastatic mucosal melanoma. BMC Cancer. 2014;14(1):516.

42. Alazzouzi $H$, Alhopuro $P$, Salovaara $R$, Sammalkorpi $H$, Järvinen $H$, Mecklin J-P, Hemminki A, Schwartz S, Aaltonen LA, Arango D. SMAD4 as a prognostic marker in colorectal cancer. Clin Cancer Res. 2005;11(7): 2606-11.

43. Li X, Liu B, Xiao J, Yuan Y, Ma J, Zhang Y. Roles of VEGF-C and SMAD4 in the lymphangiogenesis, lymphatic metastasis, and prognosis in colon cancer. J Gastrointest Surg. 2011;15(11):2001.

44. Jiang Y, Qiu Y, Minn AJ, Zhang NR. Assessing intratumor heterogeneity and tracking longitudinal and spatial clonal evolutionary history by next-generation sequencing. Proc Natl Acad Sci. 2016;113(37):5528-37.

45. Turajlic S, Xu H, Litchfield K, Rowan A, Horswell S, Chambers T, O'Brien T, Lopez Jl, Watkins TB, Nicol D, et al. Deterministic evolutionary trajectories influence primary tumor growth: Tracerx renal. Cell. 2018;173(3):595-610.

46. Turajlic S, Xu H, Litchfield K, Rowan A, Chambers T, Lopez JI, Nicol D, O'Brien T, Larkin J, Horswell S, et al. Tracking cancer evolution reveals constrained routes to metastases: Tracerx renal. Cell. 2018:173(3):581-94.

47. Abbosh C, Birkbak NJ, Wilson GA, Jamal-Hanjani M, Constantin T, Salari R, Le Quesne J, Moore DA, Veeriah S, Rosenthal R, et al. Phylogenetic ctdna analysis depicts early-stage lung cancer evolution. Nature. 2017;545(7655):446

48. Nik-Zainal S, Van Loo P, Wedge DC, Alexandrov LB, Greenman CD, Lau KW Raine K, Jones D, Marshall J, Ramakrishna M, et al. The life history of 21 breast cancers. Cell. 2012;149(5):994-1007.

49. Gillies RJ, Verduzco D, Gatenby RA. Evolutionary dynamics of carcinogenesis and why targeted therapy does not work. Nat Rev Cancer 2012;12(7):487-93

50. Burrell RA, McGranahan N, Bartek J, Swanton C. The causes and consequences of genetic heterogeneity in cancer evolution. Nature. 2013;501(7467):338-45

51. Vogelstein B, Papadopoulos N, Velculescu VE, Zhou S, Diaz LA, Kinzler KW. Cancer genome landscapes. Science. 2013;339(6127):1546-58.

52. Sottoriva A, Spiteri I, Piccirillo SG, Touloumis A, Collins VP, Marioni JC, Curtis C, Watts C, Tavaré S. Intratumor heterogeneity in human glioblastoma reflects cancer evolutionary dynamics. Proc Natl Acad Sci. 2013;110(10):4009-14.

53. Edmonds J. Optimum branchings. Math Decis Sci, Part. 1968;1:335-45.

54. El-Kebir M, Oesper L, Acheson-Field H, Raphael BJ. Reconstruction of clonal trees and tumor composition from multi-sample sequencing data. Bioinformatics. 2015:31(12):62-70.

55. Popic V, Salari R, Hajirasouliha I, Kashef-Haghighi D, West RB, Batzoglou S Fast and scalable inference of multi-sample cancer lineages. Genome Biol. 2015;16(1):795-17.

56. Chow C, Liu C. Approximating discrete probability distributions with dependence trees. IEEE Trans Inf Theory. 1968;14(3):462-7.

\section{Ready to submit your research? Choose BMC and benefit from}

- fast, convenient online submission

- thorough peer review by experienced researchers in your field

- rapid publication on acceptance

- support for research data, including large and complex data types

- gold Open Access which fosters wider collaboration and increased citations

- maximum visibility for your research: over $100 \mathrm{M}$ website views per year

At BMC, research is always in progress.

Learn more biomedcentral.com/submissions 